\title{
Review: The Role of Intestinal Dysbiosis in Parkinson's Disease
}

\section{OPEN ACCESS}

Edited by:

Maria Cecilia Giron, University of Padua, Italy

Reviewed by: Hemraj B. Dodiya,

University of Chicago, United States Carolina Pellegrini,

University of Pisa, Italy

*Correspondence: Ling Long

linglinglively@126.com; long/3@mail.sysu.edu.cn Xiaodong Cai

baggio_cxd@126.com;

caixd3@mail.sysu.edu.cn

${ }^{\text {t}}$ These authors have contributed equally to this work

Specialty section: This article was submitted to Microbiome in Health and Disease,

a section of the journal Frontiers in Cellular and Infection Microbiology

Received: 08 October 2020 Accepted: 06 April 2021 Published: 22 April 2021

Citation:

Huang Y, Liao J, Liu X, Zhong Y, Cai $X$ and Long L (2021) Review:

The Role of Intestinal Dysbiosis in Parkinson's Disease. Front. Cell. Infect. Microbiol. 11:615075. doi: 10.3389/fcimb.2021.615075

\author{
Yiying Huang ${ }^{1 \dagger}$, Jinchi Liao ${ }^{1 \dagger}$, Xu Liu ${ }^{1}$, Yunxiao Zhong ${ }^{1}$, Xiaodong Cai ${ }^{2 *}$ and Ling Long ${ }^{1 *}$ \\ ${ }^{1}$ Department of Neurology, Third Affiliated Hospital, Sun Yat-Sen University, Guangzhou, China, ${ }^{2}$ Department of Neurology, \\ Sixth Affiliated Hospital, Sun Yat-Sen University (Guangdong Gastrointestinal and Anal Hospital), Guangzhou, China
}

Several studies have highlighted the roles played by the gut microbiome in central nervous system diseases. Clinical symptoms and neuropathology have suggested that Parkinson's disease may originate in the gut, which is home to approximately 100 trillion microbes. Alterations in the gastrointestinal microbiota populations may promote the development and progression of Parkinson's disease. Here, we reviewed existing studies that have explored the role of intestinal dysbiosis in Parkinson's disease, focusing on the roles of microbiota, their metabolites, and components in inflammation, barrier failure, microglial activation, and $\alpha$-synuclein pathology. We conclude that there are intestinal dysbiosis in Parkinson's disease. Intestinal dysbiosis is likely involved in the pathogenesis of Parkinson's disease through mechanisms that include barrier destruction, inflammation and oxidative stress, decreased dopamine production, and molecular mimicry. Additional studies remain necessary to explore and verify the mechanisms through which dysbiosis may cause or promote Parkinson's disease. Preclinical studies have shown that gastrointestinal microbial therapy may represent an effective and novel treatment for Parkinson's disease; however, more studies, especially clinical studies, are necessary to explore the curative effects of microbial therapy in Parkinson's disease.

Keywords: Parkinson's disease, intestinal dysbiosis, $\alpha$-synuclein, inflammation, molecular mimicry

\section{INTRODUCTION}

Parkinson's disease (PD) is the second-most common neurodegenerative disease, after Alzheimer's disease. Approximately seven to ten million PD patients have been diagnosed worldwide (Nair et al., 2018), and this population is expected to double by 2030 (Dorsey et al., 2007). PD primarily presents as motor dysfunction including tremor, rigidity, postural instability, and bradykinesia. The nonmotor symptoms of PD include anosmia, sleep disturbance, anxiety, depression, orthostatic hypotension, and gastrointestinal (GI) symptoms. GI symptoms such as dyspepsia, hypersalivation, constipation, defecatory dysfunction, fecal incontinence, nausea, and abdominal pain usually precede motor symptoms (Cersosimo et al., 2013; Mukherjee et al., 2016). Constipation is a major intestinal dysfunction associated with PD and represents one of the earliest biomarkers of the pathologic process that ultimately results in PD development (Kalia and Lang, 2015). Constipation and intestinal inflammation often occur years before the onset of motor symptoms (Braak et al., 2003; Verbaan et al., 2007; Devos et al., 2013; Kalia and Lang, 2015). The primary pathological features of PD include the progressive loss of dopaminergic neurons in the substantia 
nigra ( $\mathrm{SN}$ ) and the formation of cytoplasmic inclusions comprising abnormal, aggregating $\alpha$-synuclein $(\alpha \operatorname{Syn})$ proteins known as Lewy bodies. $\alpha$ Syn oligomers are deposited not only in the SN but also in the brainstem, olfactory bulb, and GI tract (Punsoni et al., 2019), and Braak hypothesized that abnormal $\alpha$ Syn accumulates in the gut and spreads to the brain in a prionlike manner through the vagus nerve (Del Tredici and Braak, 2008). This view is supported by pathophysiological evidence that revealed the early appearance of $\alpha$ Syn-containing inclusion bodies in the enteric nervous system (ENS), the glossopharyngeal nerve, and the vagus nerve (Braak et al., 2003; Shannon et al., 2012), and the risk of PD development was shown to be reduced among individuals with vagus nerve amputations (Svensson et al., 2015; Liu et al., 2017). Recent studies have further confirmed Braak's proposal. When PFFs (the preformed $\alpha$ Syn fibrils)were injected into the myenteric plexus of pylorus and duodenal, pSer129- $\alpha$ Syn (phosphorylated on serine 129- $\alpha$ Syn) immunoreactivity could be detected in wild-type mice and BAC (Bacterial Artificial Chromosome) rats (the transgenic rat model overexpresses the complete human SNCA gene in its wild-type form) at the original injection site, dorsal motor nucleus of the vagus nerve and substantia nigra pars compacta, while it could only be detected in truncal vagotomy animals at the original injection site, but not in substantia nigra (Kim et al., 2019; Van Den Berge et al., 2019). Because PD appears to display pathological and clinical manifestations of the GI tract earlier than typical PD symptoms and severing the connections between the GI tract and the central nervous system (CNS) has been shown to reduce PD risk, it's reasonable to assume that local factors in the GI tract may contribute to PD development. With an area half the size of a badminton field (Helander and Fandriks, 2014), the digestive tract is susceptible to intestinal dysbiosis, as the digestive tract serves as the body's primary microorganism reservoir (Sender et al., 2016b). This dysbiosis may lead to local inflammation, pathological changes, and intestinal symptoms.

\section{INTESTINAL DYSBIOSIS AND BRAIN HEALTH}

The majority of the human microbiome is concentrated in the gut, which contains more than 10 trillion cells and up to 1,000 different microbial species (Sender et al., 2016a). The term "microbiome" refers to the genetic material (genome) contained in all microbiota found in the GI system, representing a collection of bacteria, viruses, archaea, fungi, and protozoa (Lozupone et al., 2012). Gut microbiota is a general term for all symbionts living in the gut (Adak and Khan, 2019). Most studies to date examining intestinal microorganisms focused on intestinal bacteria. Intestinal bacteria are mainly divided into seven branches, including Firmicutes, Bacteroidetes, Actinobacteria, Fusobacteria, Proteobacteria, Verrucomicrobia and Cyanobacteria, and the number of Bacteroidetes and Firmicutes accounts for more than 90\% (Adak and Khan, 2019). Intestinal dysbiosis is defined as the disruption of the "normal" balance between GI microbiota and the host
(Lozupone et al., 2012). Intestinal dysbiosis has been associated with obesity (Turnbaugh et al., 2008; Just et al., 2018), malnutrition (Ghosh et al., 2014), diabetes (Giongo et al., 2011; Karlsson et al., 2013), cardiovascular disease (Cho et al., 2017), autistic spectrum disorders (Parracho et al., 2005) and Alzheimer's disease (Vogt et al., 2017; Sochocka et al., 2019). In recent decades, a deeper understanding of GI microbiota populations has greatly complemented the concept of the gut-brain axis, which has evolved into the concept of the microbiota-gut-brain axis. Microbiota and the brain communicate with each other via various routes including the immune system, tryptophan metabolism, the vagus nerve, and the ENS through microbial metabolites such as short-chain fatty acids (SCFAs), branchedchain amino acids, and peptidoglycans (Cryan et al., 2019). GI microbiota are essential for maintaining a healthy nervous system. On the one hand, GI microbiota play roles in basic neurogenerative processes such as the formation of the blood-brain-barrier (BBB), myelination, neurogenesis, and microglia maturation (Sharon et al., 2016), as demonstrated by germ-free (GF) mice, which develop abnormal hypothalamic-pituitary-adrenal axes, changes in hippocampal nerves, reduced mRNA and protein levels of hippocampal brain-derived nerve growth factor (BNGF), and impaired BBB function (Sudo et al., 2004; Braniste et al., 2014; Mohle et al., 2016). On the other hand, GI microbiota are highly important to the immune system (Rooks and Garrett, 2016), as they control microglial maturation and its function (Erny et al., 2015), and GF mice show immunodeficiencies at both the cellular and structural levels (Round and Mazmanian, 2009). When colonizing human bodies, various microbes provide an abundance of antigens, which are essential for the healthy maturation of the immune system during early life (Cahenzli et al., 2013). It is worth emphasizing that microbial homeostasis is crucial to individual health.

It has been proved that intestinal dysbiosis can promote the pathology of PD. Compared with mice exposed to lipopolysaccharide (LPS) negative bacteria, mice injected with $\alpha$ Syn and exposed to LPS positive bacteria produced a unique form of $\alpha$ Syn fibers. This abnormal protein aggregation may further form the typical pathological changes of PD (Fitzgerald et al., 2019). In another study, the mRNA level of $\alpha$ Syn in SHSY5Y cells (human neuroblastoma) treated with B. mirabilis was significantly higher than that in the untreated cells. This means that there may be an increase in the production of $\alpha$ Syn. They further investigated the differences in the aggregation of $\alpha$ Syn: on day 16 after administration of M. mirabilis, $\alpha$ Syn filaments in the distal colon increased significantly, and in the $\mathrm{SN}$, the immunoreactivity of $\alpha$ Syn filaments was significantly stronger than that of normal group (Choi et al., 2018).

\section{ALTERED GASTROINTESTINAL MICROBIOTA COMPOSITION IN PD}

Intestinal dysbiosis has been proposed to act as a trigger or enabler of PD. In mice that overexpress $\alpha$ Syn, GI microbiota aggravates the expression of motor deficits, microglial activation, and $\alpha$-Syn 
pathology. The deficits reduce but don't disappear entirely in the absence of microbiota (Sampson et al., 2016). The chronic oral administration of rotenone has been reported to cause GI dysfunction and intestinal dysbiosis prior to the development of motor dysfunction and CNS pathology (Yang et al., 2017). Now that animal experiments have confirmed that intestinal dysbacteriosis plays a role in $\mathrm{PD}$, we have to understand what changes have taken place in the intestinal microbiota.

Various clinical studies have shown evidence indicating the occurrence of intestinal dysbiosis in PD patients compared with healthy controls, and the compositions of both fecal and mucosal microorganisms have been reported to change in PD patients (Hasegawa et al., 2015; Keshavarzian et al., 2015; Unger et al., 2016). These differences become particularly pronounced at the family, genus, and operational taxonomic unit (OTU) levels. At the taxonomic level, butyrate-producing bacteria were reported to be much more abundant in fecal samples from controls than in those from PD patients (Keshavarzian et al., 2015). At the genomic level, the expression levels of several genes associated with metabolism were significantly reduced in the PD fecal microbiome, whereas gene expression associated with LPS biosynthesis and type III bacterial secretion systems was significantly increased in PD patients (Keshavarzian et al., 2015). One study reported that the putative cellulose-degrading bacteria, from the genera Blautia, Faecalibacterium, and Ruminococcus, were significantly decreased, whereas the putative pathobionts from the genera Escherichia-Shigella Streptococcus Proteus, and Enterococcus were significantly increased, in PD subjects compared with healthy controls. Correlation analysis indicated that disease severity and PD duration were negatively correlated with the presence of putative cellulose degraders and positively correlated with the presence of putative pathobionts (Li et al., 2017). Here, we summarized several studies to illustrate changes that have been described in the intestinal microbiota populations of $\mathrm{PD}$ patients (see Table 1).

\section{Helicobacter pylori (H. pylori)}

Helicobacter pylori have been associated with PD since the 1960 s (Hashim et al., 2014). A meta-analysis suggested that H. pylori infections might be associated with PD risk (Shen et al., 2017). When compared to $H$. pylori-negative PD patients, $H$. pyloriinfected PD patients present with worse motor dysfunction and they display a 12 -fold increase in mortality rate (McGee et al., 2018; Zendehdel and Roham, 2020). Additionally, H. pylori may affect the absorption of levodopa, and the eradication of $H$. pylori in PD patients has been shown to improve motor function and levodopa absorption compared with those in PD patients in whom H. pylori were not eradicated (McGee et al., 2018). Besides, $H$. pylori eradication not only extended ON duration but also improved the levodopa onset time and quality of life parameters (Hashim et al., 2014). However, a recent study has shown that $H$. pylori eradication did not improve the MDSUPDRS scores of PD patients significantly, whether it is part of motor, nonmotor or quality of life outcome (Tan et al., 2020). More well-designed trials are needed to verify the effect of H. pylori eradication in PD.

\section{Prevotellaceae}

Most studies have consistently reported reduced Prevotellaceae populations in PD patients (Bedarf et al., 2017; Gerhardt and Mohajeri, 2018; Aho et al., 2019; Lin et al., 2019; Vidal-Martinez et al., 2020), and Prevotellaceae populations have been significantly correlated with PD severity scores (Jin et al., 2019). Prevotellaceae are symbiotic bacteria that are involved in the synthesis of neuroactive SCFAs (such as acetate, propionate, and butyrate) through fiber fermentation, and the release of thiamine and folate. The alteration of butyrate concentration affects the expression of occludin, a component of tight junction protein, and this alteration may further affect the intestinal permeability (Wang et al., 2012). Increased levels of

TABLE 1 | Changes in the intestinal microbiota populations of patients of Parkinsons's disease.

\begin{tabular}{|c|c|c|c|c|}
\hline Phylum & Family & Genus & Change & Reference \\
\hline Actinobacteria & Bifidobacetriaceae & Bifidobaterium & $\uparrow$ & (Unger et al., 2016; Hill-Burns et al., 2017; Petrov et al., 2017; Vidal-Martinez et al., 2020) \\
\hline \multirow[t]{2}{*}{ Bacteroidetes } & Prevotellaceae & Prevotella & $\downarrow$ & (Hill-Burns et al., 2017; Lin et al., 2019; Vidal-Martinez et al., 2020) \\
\hline & Bacteroidaceae & Bacteroides & $\downarrow$ & (Hasegawa et al., 2015) \\
\hline \multirow[t]{12}{*}{ Firmicutes } & Lachnospiraceae & & $\downarrow$ & (Lin et al., 2018; Jin et al., 2019) \\
\hline & & Blautia & $\downarrow$ & (Hill-Burns et al., 2017) \\
\hline & & Roseburia & $\downarrow$ & (Aho et al., 2019) \\
\hline & Ruminococcaceae & Ruminococcus & $\downarrow$ & (Li et al., 2017) \\
\hline & Eubacteriaceae & Eubacterium & $\downarrow$ & (Keshavarzian et al., 2015) \\
\hline & Enterococcaceae & & $\uparrow$ & (Hopfner et al., 2017) \\
\hline & & Enterococcus & $\uparrow$ & (Li et al., 2017) \\
\hline & Lactobacillaceae & & $\uparrow$ & (Hopfner et al., 2017; Lin et al., 2018) \\
\hline & & Lactobacillus & $\uparrow$ & (Bedarf et al., 2017; Lin et al., 2019; Baldini et al., 2020) \\
\hline & Christensenellaceae & Christensenella & $\uparrow$ & (Petrov et al., 2017; Baldini et al., 2020) \\
\hline & Clostridiaceae & Clostridium XVIII & $\uparrow$ & (Qian et al., 2018) \\
\hline & & Clostridium IV & $\uparrow$ & (Qian et al., 2018) \\
\hline Proteobacteria & Enterobacteriaceae & & $\uparrow$ & (Unger et al., 2016) \\
\hline \multirow[t]{2}{*}{ Verrucomicrobia } & Verrucomicrobiaceae & Verrucomicrobia & $\uparrow$ & (Lin et al., 2019) \\
\hline & & Akkermansia & $\uparrow$ & $\begin{array}{l}\text { (Keshavarzian et al., 2015; Bedarf et al., 2017; Hill-Burns et al., 2017; Lin et al., 2019; } \\
\text { Vidal-Martinez et al., 2020) }\end{array}$ \\
\hline
\end{tabular}


bacterial endotoxins (such as LPS) due to increased intestinal permeability can lead to the overexpression and aggregation of $\alpha$ Syn, which is essential during the development of PD (Forsyth et al., 2011; Sergeyeva and Sergeyev, 2011; Peralta Ramos et al., 2019). Additionally, decreased Prevotellaceae levels can lead to the potential development of $\alpha$-synucleinopathies by reducing the concentration of SCFAs, which leads to the destruction of intracellular and extracellular protein clearance mechanisms associated with SCFA-dependent gene expression (De Vadder et al., 2014).

\section{Enterobacteriaceae}

Enterobacteriaceae have been reported to be more abundant in fecal samples from PD patients compared with matched controls (Unger et al., 2016). Compared with the tremor-dominant (TD) PD subtype, the contents of Enterobacteriaceae bacteria are richer in patients with postural instability and gait difficulty (PIGD)-type PD (Scheperjans et al., 2015). The relative abundance of Enterobacteriaceae was positively associated with the severity of PIGD-type PD (Scheperjans et al., 2015).

\section{Eubacteriaceae}

Several studies have found that the intestinal Eubacteria population decreases in PD patients compared with controls (Keshavarzian et al., 2015; Bedarf et al., 2017). Eubacteria have been associated with the reduced gene expression necessary for the degradation of 5-dihydro4-deoxy-D-glucuronate and two additional tryptophan metabolism pathways associated with increased formate conversion (Bedarf et al., 2017). Glucuronidase enzymes mediate not only the regeneration of molecules important for host health but also the generation of toxins and carcinogens and the activation of endogenous glucuronides such as hormones and neurotransmitters (Gerhardt and Mohajeri, 2018). Rotenone-induced male PD-model Wistar rats demonstrate significant decreases in mean tryptophan and serotonin levels over a 24-h period (Mattam and Jagota, 2015). Serotonin synthesis requires tryptophan as the substrate, and the increased activity of other tryptophan metabolic pathways accelerates the consumption of tryptophan, which results in the decreased synthesis of serotonin, and serotonin deficiency has been associated with the pathophysiological process of PD (Weng et al., 2016).

\section{EFFECTS OF BACTERIAL METABOLITES AND COMPONENTS ON PD}

The GI tract is an important location for the host immune system to communicate with microbes, and healthy individuals maintain a balance between tolerance for commensals and immunity against pathogens. During intestinal dysbiosis, the metabolites or components of pathogenic bacteria may cause inflammation, combined with metabolic changes in the symbiotic bacteria that regulate inflammation, which, together, result in $\mathrm{PD}$ pathology.

\section{SCFAs}

SCFAs are the major products of microbial fermentative activity in the gut, including acetate, propionate, and butyrate
(Cummings et al., 1987). Compared with healthy controls, PD patients contained fewer members of the family Lachnospiracae (Hill-Burns et al., 2017; Jin et al., 2019) and the genus Faecalibacterium (Keshavarzian et al., 2015; Unger et al., 2016), two kinds of probiotics producing SCFAs, in their intestinal tracts. Consistent with this finding, current studies have reported decreased fecal concentrations of SCFAs (Perez-Pardo et al., 2018).

Butyrate is the preferred energy source for colon cells and is locally consumed (Koh et al., 2016). Reduced SCFA levels in the colon may reduce colon movement, leading to constipation in PD. One study concluded that butyrate increases colonic transit and neuronally mediated contractile responses in the colon (Soret et al., 2010).

SCFAs are associated with increased intestinal permeability. On one hand, SCFAs are generally regarded as active microbial metabolites, with anti-inflammatory properties (Chang et al., 2014; Koh et al., 2016), and studies have suggested that the increased intestinal permeability to endotoxins among PD patients is caused by microbial intestinal inflammation (PerezPardo et al., 2018; Perez-Pardo et al., 2019). SCFAs activate Gprotein-coupled receptor (GPR)41 [also known as free-fatty acid receptor (FFAR)3] and GPR43 (FFAR2) on intestinal epithelial cells, which leads to mitogen-activated protein kinase (MAPK) signaling and the rapid production of chemokines and cytokines, which mediate protective immunity and tissue inflammation in mice (Kim et al., 2013). On the other hand, butyrate affects the expression of the tight junction proteins claudin 1 and claudin 2, and butyrate supplementation contributes to the integrity of the intestinal barrier (Ploger et al., 2012; Wang et al., 2012).

One study reported that SCFAs regulate microglial homeostasis (Erny et al., 2015). In this study, GF mice showed altered microglial gene expression, protein production, and morphology, similar to those observed during immature or dysfunctional states; however, these defective microglia could be restored by SCFA supplementation delivered through the drinking water for 4 weeks. SCFAs regulate the maturation of microglia by enabling the microglial expression of SCFAresponsive genes such as histone deacetylase.

SCFAs enhance the integrity of the BBB. A mouse study showed that after treatment with single bacterial strain Clostridium tyrobutyricum (which primarily produces butyrate), Bacteroides thetaiotaomicron (which produces primarily acetate and propionate), or SCFAs, the adult GF mice presented with decreased $\mathrm{BBB}$ permeability and the increased expression of occludin in the frontal cortex, hippocampus, and striatum (Braniste et al., 2014).

However, SCFAs have also been reported to promote $\alpha$ Synmediated neuroinflammation and motor deficits. After SCFAs administration, GF mice were found with $\alpha$ Syn aggregation and motor deficits, although SCFAs did not induce $\alpha$ Syn aggregation and structural changes of $\alpha$ Syn amyloid fibrils in vitro (Sampson et al., 2016). This is contrary to the anti-inflammatory properties of SCFAs mentioned above. It is possible that SCFAs play an extremely complex role in the pathological progress and symptoms of $\mathrm{PD}$, but we have not fully understood it. At the 
same time, GF animals are widely used in the study of gut microbiota and PD, but have we considered that GF animals have immune deficiency?

\section{LPS}

LPS is a component in the cell walls of Gram-negative bacteria. Bacterial genes involved in LPS biosynthesis were increased in mucosal and fecal samples from PD patients compared with those in non-PD patients, and serum lipopolysaccharide-binding protein (LBP) levels in $\mathrm{PD}$ patients were lower than those of controls when the integrity of the intestinal mucosa was not compromised (Hasegawa et al., 2015). Clinical studies have confirmed that LBP is a GI marker of LPS induced neurotoxicity, but its level has nothing to do with the severity of PD (Pal et al., 2015). Although the serum endotoxin level did not increase significantly, the LBP level decreased significantly, compared with the controls, which also proved that there was inflammation caused by increased endotoxin (Forsyth et al., 2011). These findings indicated the increased contact with serum bacteria and/or LPS and that the bacterial products are likely to reach the CNS, causing neuroinflammation.

Toll-like receptor (TLR) can be found in the immune system and epithelial tissue, and the neurons and glial cells of the peripheral and CNS. These receptors specifically recognize evolutionarily conserved molecular structures, known as pathogen-associated molecular patterns (PAMPs), which are widely expressed by various infectious microorganisms. Additionally, TLRs are associated with damage-associated molecular patterns (DAMPs), a group of endogenous molecules that are produced during tissue damage. TLRs can be divided into many subfamilies, based on the types of PAMPs and DAMPs that they identify. LPS, for example, is primarily recognized by TLR4.

LPS has been associated with both systemic and CNS inflammation. The combination of LPS and TLR4 recruits Toll/interleukin-1 receptor (TIR) domain-containing adaptors such as myeloid differentiation primary response gene 88 (MyD88) or the TIR-domain-containing adapter-inducing interferon- $\beta$ (TRIF) pathway to deliver signals to several downstream pathways, including nuclear factor-kappa B (NF-KB), MAPKs, and/or interferon-regulatory factor signaling pathways, which are pivotal for the expression of various gene products involved in the inflammatory responses (Kawasaki and Kawai, 2014; Caputi and Giron, 2018). Intra-cerebral LPS administration can induce localized microgliosis and microglial activation at the site of the injection (Liu and Bing, 2011; Hoban et al., 2013; Flores-Martinez et al., 2018). Moreover, some studies have found increased levels of proinflammatory cytokines, including interleukin (IL)-1 $\beta$, tumor necrosis factor-alpha (TNF- $\alpha$ ), IL-6, and nitric oxide (NO) in the SN, after LPS injection (Choi et al., 2010; Liu and Bing, 2011; FloresMartinez et al., 2018). The body temperatures of rats injected with LPS gradually increased to a maximum of $38.25 \pm 0.15^{\circ} \mathrm{C}$, and adynamia and a curled body posture were also observed (Flores-Martinez et al., 2018).

LPS also induces progressive SN dopaminergic neurodegeneration and decreased striatal dopamine levels (Hunter et al., 2009; Liu and
Bing, 2011; Hoban et al., 2013). However, a 36-day mouse study indicated that LPS exposure induced progressive dopaminergic neuronal loss, starting after day 5 and lasting through day 19, after which no additional significant cell loss was observed (Beier et al., 2017).

LPS induces nitrosative and oxidative stress. Nitrite concentrations, as a marker of nitrosative stress, and malondialdehyde (MDA) and 4-hydroxyalkenals (4HAE) levels, as a marker of oxidative stress, were significantly increased following the LPS injection (Flores-Martinez et al., 2018). LPS can enhance the expression of inducible NO synthase (iNOS) and promote the nitration and polymerization of $\alpha$ Syn. iNOS-derived $\mathrm{NO}$ is important for the progression of LPS-induced dopaminergic neurodegeneration. At 4 weeks after injection, NOS inhibitors [LN6-(1-iminoethyl)-lysine (L-NIL) and nomega-nitro-L-arginine (L-NNA)] attenuated LPS-induced tyrosine hydroxylase (TH)positive cell loss, and iNOS knockout mice displayed a $30 \%$ increase in TH-positive cells compared with wild-type (WT) mice, 2 weeks after injection (Hunter et al., 2009).

\section{Amyloid Protein Curli}

Curli fibers represent a class of functional amyloid fibers that are produced by Escherichia coli (E.coli) and other Gram-negative bacteria and serve as protein scaffolds in the biofilm extracellular matrix (ECM), where they account for $85 \%$ of the total biofilm ECM (Klein et al., 2018). Two divergently transcribed operons, csgBAC and csgDEFG, encode proteins associated with the Curli structure and biogenesis (Klein et al., 2018). Curli fibers primarily comprise CsgA. As long fibers, Curli fibers extend from the cell surface, using a specific and highly controlled pathway of assembly, known as nucleation-precipitation and sometimes referred to as the type VIII secretion system, to avoid the formation of oligomeric intermediates cytotoxicity induction (Blanco et al., 2012; Klein et al., 2018; Miraglia and Colla, 2019).

Unlike pathogenic amyloid proteins such as $\beta$-amyloid 1-42 and $\alpha$ Syn, Curli is a type of functional amyloid protein, which forms biofilms and enhances bacterial adhesion and colonization (Miraglia and Colla, 2019). Intriguingly, Curli can also enhance the intestinal barrier function. Epithelial cells directly respond to curli fibers, which leads to barrier reinforcement and a reduction in the level of bacterial translocation. Additionally, on the other side of the barrier, immune cells responded to Curli, which caused the expression of IL-17 and IL-22, which have been shown to regulate barrier function in a TLR2-dependent manner (Tursi and Tükel, 2018). But more importantly, Curli can trigger systemic inflammation by binding fibronectin, plasminogen, and tissue-type plasminogen activator, as well as fibrinogen and bradykinin, which facilitate bacterial dissemination. As a PAMP, Curli binds and activates TLR2, which causes the production of pro-inflammatory cytokines and chemokines such as IL-8, IL- 6 , TNF- $\alpha$, and NO. The Curlimediated activation of TLR2 leads to the activation of the NOD-, LRR- and pyrin domain-containing protein 3 (NLRP3) inflammasome following the activation of caspase-1/11 and the maturation of pro-IL-1 $\beta$ to IL-1 $\beta$ (Tursi and Tükel, 2018; Miraglia and Colla, 2019). Extracellular misfolded fibrillar $\alpha$ Syn is also recognized as a PAMP or DAMP by microglial 
TLR2 (Caputi and Giron, 2018), which triggers an inflammatory response. This suggests that human $\alpha$ Syn might be recognized as a microbe-associated molecular pattern (MAMP) that mimics bacterial amyloids (Miraglia and Colla, 2019).

Curli subunits enhance PD progression. Bacterial and host amyloid precursor peptides fold into highly conserved, betasheet structures because of the presence of conversed glutamine and asparagine residues (Tursi and Tükel, 2018), therefore, some of the mediators involved in bacterial amyloid biogenesis may also affect the structure and function of host amyloids. Two $E$. coli genes in the Curli operon encode the chaperone-like proteins $\mathrm{CsgC}$ and $\mathrm{CsgE}$, which can both reduce in vitro amyloid formation mediated by CsgA (Chorell et al., 2015). CsgC inhibits the primary nucleation and/or elongation of CsgA through a series of electrostatic interactions, which leaves CsgA in an amorphous, $\beta$-strand-deficient state within the periplasmic space, to prevent host cell toxicity (Klein et al., 2018). The accessory periplasmic protein $\mathrm{CsgE}$ acts as a gating and specificity factor for the CsgG pore during the transport of Curli structural components (Klein et al., 2018). Chorell and his colleagues reported that the inhibitory effects of CsgC arise because of transient interactions that promote the formation of spherical $\alpha$ Syn oligomers. They also reported that CsgE accelerates $\alpha$ Syn amyloid formation through transient contacts (Chorell et al., 2015). The interactions between the C-terminal domain and the non-amyloid-component (NAC) region of $\alpha$ Syn are important for the prevention of $\alpha$ Syn fibrillization. CsgE may promote the aggregation of $\alpha$ Syn by releasing the intra-protein interactions between the C-terminal domain and the NAC region, whereas $\mathrm{CsgC}$ strengthens this interaction, which may inhibit $\alpha$ Syn aggregation (Tursi and Tükel, 2018). In rat model experiments, the repeated oral administration of Curliproducing bacteria resulted in the accumulation of intestinal $\alpha$ Syn deposits in aging WT animals; furthermore, microgliosis and astrogliosis and elevated levels of TLR2, IL-6, and TNF were also found in the brain. In $\alpha$ Syn-overexpressing animals, which received repeated oral administrations of Curli-producing bacteria, increased neuronal $\alpha$ Syn deposition in both the gut and the brain tissues were reported (Miraglia and Colla, 2019).

\section{POSSIBLE MECHANISMS THROUGH WHICH INTESTINAL DYSBIOSIS CAUSES PD}

\section{Increased Permeability of the Intestinal Barrier and the BBB}

Alterations in intestinal microbiota populations can increase intestinal barrier permeability. The changes of intestinal permeability in PD patients have been reported. The decreased expression level of colonic occludin and the abnormal subcellular distribution of occludin and ZO-1 have suggested that the change of intestinal permeability in PD patients (van and Derkinderen, 2019). Furthermore, compared to controls, increased 24-hour urinary excretion of sucraloses has suggested increased intestinal permeability in PD subjects (Forsyth et al., 2011). Reduced concentrations of intestinal SCFAs contribute to the increased intestinal mucosal permeability (see above). Akkermansia degrades the mucous layer, using the mucus for energy, and the increased population of this genus leads to the increased permeability of the intestinal mucous barrier (Forsyth et al., 2011; Keshavarzian et al., 2015; Bedarf et al., 2017; Hill-Burns et al., 2017).

The permeability of the BBB can be altered according to changes in the intestinal microbiota population. Tight junctions maintain normal BBB permeability. Compared with normal pathogen-free mice, the expression levels of claudin and claudin 5 in the brains of GF mice were decreased, which was associated with increased BBB permeability (Braniste et al., 2014). The administration of sodium butyrate or normal flora, derived from pathogen-free mice, into GF mice induced the increased expression of claudin and the decreased permeability of the BBB (Braniste et al., 2014). There are intestinal dysbiosis in PD patients, the altered microbes may lead to change of BBB permeability.

\section{Inflammation and Oxidative Stress}

Microglial activation, astrocyte proliferation, and lymphocyte infiltration can be observed in the $\mathrm{SN}$ and striatum of $\mathrm{PD}$ patients, in addition to the increased secretion of inflammatory factors (Hirsch and Hunot, 2009). We have summarized decreased SCFAs level and increased synthesis of LPS in PD patients above. SCFAs promote the maturation and activation of microglia (Erny et al., 2015) and decreased SCFA concentrations in the intestinal cavity depress anti-inflammatory activities, which supports local and systemic inflammation. The injection of LPS into the striatum and globus pallidum of mice resulted in the activation of microglia, the development of a proinflammatory M1 phenotype at both the original injection site and in the $\mathrm{SN}$, and the loss of dopaminergic neurons (BaizabalCarvallo and Alonso-Juarez, 2020). In the CNS and peripheral nervous system, LPS binds to TLR4 on microglia, which activates microglia. Increased levels of NLRP3 and IL-1 $\beta$ have been found in the central and peripheral nervous system (Pellegrini et al., 2020). There may be such inflammatory factor activation pathways: LPS binds to TLRs, promoting pro-IL-1 $\beta$ and NLRP3 transcription by activating nuclear factor $-\kappa B(N F-\kappa B)$ via the pathway of myeloid differentiation primary-response 88 (MyD88) or the receptor- interacting protein 1 (RIP1)-FASassociated death domain protein (FADD)-caspase- 8 protein complex. Moreover, LPS may stimulate TLR4 to activate RIP1FADD-caspase- 8 protein complex and directly activate typical NLRP3 oligomerization and assembly. The oligomerization of NLRP3 inflammasome leads to the activation of caspase- 1 and the subsequent release of IL-1 $\beta$ and IL-18 (Pellegrini et al., 2020). In the blood circulation, LPS activates mononuclear cells, and both LPS-induced pathways result in the release of inflammatory cytokines, such as IL- $1 \beta$ and TNF- $\alpha$ (Gutsmann et al., 2001; Murakami et al., 2009). The repeated administration of Curli-producing bacteria in rats can enhance microgliosis, astrogliosis, and cytokine production (Chen et al., 2016). Additionally, colon biopsies of PD patients have shown the increased expression of TLR4, CD ${ }^{+}$T-lymphocytes, and various cytokines associated with intestinal dysbiosis (PerezPardo et al., 2019). LPS triggers oxidative stress in the CNS, 
which promotes the release of $\mathrm{NO}$ by activating the expression of iNOS (see above), and excess NO promotes the nitration and polymerization of $\alpha$ Syn.

\section{Changes of the Production of Dopamine}

The GI tract produces almost half of the dopamine produced by the body (Eisenhofer et al., 1997), and dopamine can be produced by various intestinal microbiota populations (Wall et al., 2014). Dopamine produced by gut microbes may enter the bloodstream directly or influence dopamine metabolism through receptors on the ENS or other structures. Piglets given antibiotics at the distal ileum demonstrated altered microbial compositions in feces, and antibiotic infusions resulted in a significant decrease in dopamine concentrations in the hypothalamus and in the blood (Gao et al., 2018).

Furthermore, altered gut microbiota populations affected the production of dopamine by affecting the levels of the intestinal hormone ghrelin. Ghrelin is a GI hormone that specifically recognizes the endogenous ghrelin receptor, also known as growth hormone secretagogue receptor (GHSR), in the hypothalamus, which regulates food intake and obesity. Ghrelin binds to $\mathrm{SN}$ pars compacta (SNpc) cells that express GHSR to activate SNpc dopaminergic neurons, which enhances the transcription of TH mRNA and increases the concentration of dopamine in the dorsal striatum (Andrews et al., 2009). A study showed that gut microbiota metabolites such as SCFAs, lactate, and bacterial supernatants can attenuate ghrelin-mediated signaling through GHSR-1a (Torres-Fuentes et al., 2019). A probiotic preparation, Lactogen 13, has been shown to induce high ghrelin expression levels in the stomach/intestine of tilapia (Gioacchini et al., 2018). Therefore, gut microbiota can indirectly alter dopamine production by influencing the levels of ghrelin.

\section{Molecular Mimicry}

Thus far, researchers have found many proteins that share homologous amino acid sequences with $\alpha$ Syn, some of which have been shown to cross-react with $\alpha$ Syn. Autoantibodies that recognize the HSV1-U14222-36 peptide cross-react with the homologous human $\alpha$ Syn100-114 epitope, which is part of the acidic C-terminal region. Autoantibodies may affect the physiological functions of this region in vivo because the Cterminal region is the aggregation inhibition region (Ozansoy and Başak, 2013; Caggiu et al., 2016). Clone CS1-4 is a commercially-available cocktail that contains four monoclonal antibodies that recognize the C-terminus of the Epstein-Barr virus latent membrane protein 1 (LMP-1), it is able to produce intense, highly specific Lewy body labeling in the SN of postmortem PD brains. Furthermore, preclinical studies have shown that the CS1-4 antibody cocktail can induce $\alpha$ Syn aggregation and BLAST searches have revealed a similar region between the primary sequences of LMP-1 and $\alpha$ Syn, which comprises the amino acids PXDPDN (Woulfe et al., 2014). Besides, Miraglia and colleagues suggested that human $\alpha$ Syn might be recognized as an MAMP mimicking bacterial amyloids (Miraglia and Colla, 2019).

The proposed mechanisms are concluded in Figure 1.

\section{APPLICATION OF MICROBIAL THERAPY IN PD}

\section{Probiotics}

The Food and Agriculture Organization of the United Nations and the World Health Organization have defined probiotics as "live microorganisms which, when administered in adequate amounts, confer a health benefit on the host" (Hill et al., 2014). Several studies have consistently shown that taking probiotics can improve constipation symptoms in PD patients (Cassani et al., 2011; Barichella et al., 2016). In another clinical study, probiotics treatment can significantly improve abdominal pain and abdominal distension, but the effect is poor for constipation with incomplete emptying (Georgescu et al., 2016). Consistent with most research conclusions, a recent clinical study has shown that after 4 weeks of probiotics treatment, the frequency of spontaneous defecation, stool consistency, constipation severity score and constipation related quality of life in patients with PD were significantly improved (Tan et al., 2021). In an in vitro study that explored the effects of probiotics on mediators of inflammation and oxidative damage in primary blood mononuclear cells (PBMCs) from PD patients, Lactobacillus salivarius (LS01) and Lactobacillus acidophilus (LA02) significantly decreased all proinflammatory cytokines, increased anti-inflammatory cytokines, and significantly reduced reactive oxygen species production (Magistrelli et al., 2019). Furthermore, probiotics are beneficial for the prevention of pathological formations associated with $\mathrm{PD}$. The probiotic strain PXN21 inhibited $\alpha$ Syn aggregation and clear preformed aggregates in a Caenorhabditis elegans model displaying established synucleinopathy (Goya et al., 2020). In a RCT study involving 60 PD patients, significantly lower MDS-UPDRS scores and high-sensitivity C-reactive protein, as well as significantly higher glutathione levels and insulin sensitivity were found in the probiotics group compared with the placebo group after 12 weeks of administration (Tamtaji et al., 2019).

\section{Prebiotics}

The International Scientific Association for Probiotics and Prebiotics (ISAPP) defined prebiotics as "a substrate that is selectively utilized by host microorganisms conferring a health benefit" (Gibson et al., 2017). The most studied prebiotics are fructo-oligosaccharide (FOS) and galacto-oligosaccharide (GOS). Prebiotics also include inulin and SCFAs. FOS supplementation increases the frequency of defecation, improves the appearance of the stool, and increases colonic transit in elderly continuous ambulatory peritoneal dialysis (CAPD) patients (Meksawan et al., 2016). In a mouse model of PD, dietary interventions supplemented with nutrients associated with phospholipid synthesis and prebiotic fibers including GOS and FOS improved cognitive functions, reduced gastrointestinal dysfunction, and increased dopamine transporter expression. Reduced motor symptoms, colon $\alpha$ Syn deposition, and intestinal inflammation were associated with a dietary intervention that included uridine and docosahexaenoic acid (DHA) (Perez-Pardo et al., 2017).

\section{Fecal Microbiota Transplantation}

Fecal microbiota transplantation (FMT) is a type of microbial therapy that can directly alter the recipient's gut microbiota population to normalize the composition, which has therapeutic 


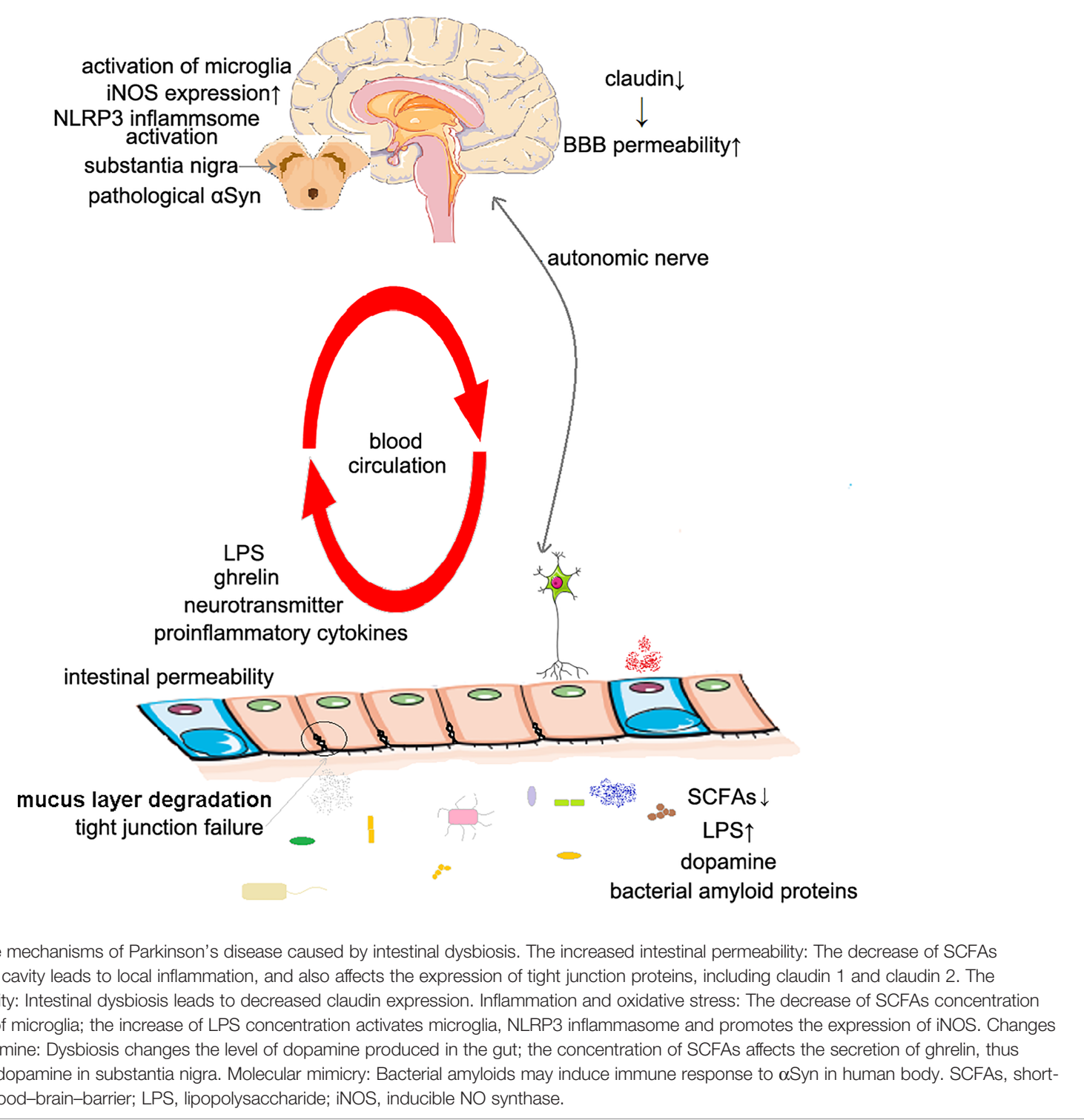

benefits. A case report showed that the time spent in defecation after transplantation was reduced from over 30 minutes to under 5 minutes, which was maintained for 3 months until the end of the follow-up period. Tremor in the legs nearly disappeared 1 week after FMT, but recurred after 2 months with reduced severity compared with that pre-FMT treatment (Huang et al., 2019). After FMT, neuroinflammation alleviation was observed in PD model mice, characterized by reduced glial activation (including both microglia and astrocytes) and the inhibition of the TLR4/TANK-binding kinase 1 (TBK1)/TNF- $\alpha$ signaling pathway (Sun et al., 2018). Increased dopaminergic neurons and TH levels in the striatum were also observed, accompanied by increased levels of dopamine, 5-hydroxytryptamine, and their metabolites.

The studies of microbial therapies of PD are presented in Table 2.

\section{CONCLUSION}

Intestinal dysbiosis has been identified in PD patients, with differences at lower classification levels, such as family, genus, and OTUs, observed between PD patients and healthy controls. The influence of gut microbiota on the CNS has not been fully elucidated. By viewing the entire intestinal microbiota population as a single genome or metabolome, large changes can be observed in the gut, manifested as decreased SCFA levels, elevated release of LPS, and alterations in the synthesis of enterogenic neurotransmitters. These microbial metabolites and components can influence the immune and nervous function of the host through various mechanisms including the increased permeability of barriers, inflammation, and oxidative stress, decreased dopamine production, and molecular mimicry. Intestinal microbiota plays an important role in the course of $\mathrm{PD}$; 
TABLE 2 | A conclusion of microbial therapy in Parkinson's disease.

\begin{tabular}{|c|c|c|c|c|c|}
\hline Therapeutic methods & Research type & $\begin{array}{l}\text { Improvement of } \\
\text { constipation }\end{array}$ & $\begin{array}{l}\text { Improvement of } \\
\text { symptoms of PD }\end{array}$ & Reduced inflammation & $\begin{array}{l}\text { Improvement of } \\
\alpha \text { Syn pathology }\end{array}$ \\
\hline \multirow[t]{2}{*}{ Probiotics } & Clinical studies & $\begin{array}{l}\text { Cassani et al., 2011; } \\
\text { Barichella et al., 2016; } \\
\text { Tan et al., } 2021\end{array}$ & Tamtaji et al., 2019 & Tamtaji et al., 2019 & \\
\hline & Preclinical studies & & & Magistrelli et al., 2019 & Goya et al., 2020 \\
\hline \multirow[t]{2}{*}{ Prebiotics } & Clinical studies & Barichella et al., 2016 & & & \\
\hline & Preclinical studies & & Perez-Pardo et al., 2017 & Perez-Pardo et al., 2017 & Perez-Pardo et al., 2017 \\
\hline \multirow[t]{2}{*}{ FMT } & Clinical studies & Huang et al., 2019 & Huang et al., 2019 & & \\
\hline & Preclinical studies & & & Sun et al., 2018 & \\
\hline
\end{tabular}

FMT, Fecal microbiota transplantation.

however, whether the microbiota population acts as a trigger or as a promoter requires further research. Since the concept of gutbrain axis was put forward, a large number of related studies have supplemented its content and promoted it to evolve into microbiota-gut-brain axis. On one hand, the microbiota- gutbrain axis is a bi-directional bioinformation network, which involves multiple communication pathways. Neurotransmitters and neuroactive substances, SCFAs, tryptophan metabolites, bioactive molecules produced by intestinal microbiota, as well as neuroactive substances produced by intestinal endocrine cells are able to enter the blood circulation, and enter the CNS to regulate brain function. In addition, the pathogenic components of intestinal microorganisms can activate the inflammatory cells in the GI tract and promote the production of inflammatory factors, which reach the CNS through blood circulation (Cryan et al., 2019). On the other hand, the brain regulates the intestinal movement and intestinal environment by regulating the function of autonomic nerve (Collins et al., 2012). The neural circuits between the gut and CNS involve afferent and efferent nerves. The ENS has been considered as a pathway for transmitting pathological synuclein. Recent animal studies have shown that pathological $\alpha$ Syn can propagate from dorsal motor nucleus of the vagus nerve to stomach, from autonomic ganglion and sympathetic trunk to cardiac sympathetic nerve after initial retrograde propagation along parasympathetic and sympathetic nerves (Van Den Berge et al., 2019). This finding further suggests the bidirectional communication characteristics of microbiotagut-brain axis from the perspective of pathology. In this article, we mainly discussed the effect of microbial dysbiosis on CNS, specifically, on PD. Indeed, from the conclusion of a large number of clinical and preclinical studies, intestinal dysbiosis has played an important role in PD. The etiology of PD is still in exploration, and there is no clear answer; intestinal dysbiosis plays a role in the progress of $\mathrm{PD}$, but the specific mechanism is still unclear. However, there have been more or less some defects in the current research. We are always getting the information of a specific time and a specific area, but we have no way to observe the dynamic evolution process and the situation of the whole body. In clinical studies, subjects' dietary habits and genetic backgrounds have not been considered sometimes. And in animal experiments, most of the subjects were young animals, while $\mathrm{PD}$ is more common in the elderly. More rigorous experimental design and more advanced detection methods need to be applied to the study of PD.

Interestingly, it has been suggested that $\mathrm{PD}$ can be divided into the peripheral nerve system (PNS)-first phenotype and the CNS-first phenotype. the PNS-first phenotype is characterized by the first sign of damage in the autonomic nervous system, and often shows rapid eye movement sleep behavior disorder (RBD) in the prodromal phase of PD; the CNS-first phenotype of SN damage precedes the autonomic nervous system, and often shows no RBD in the prodromal phase of PD (Borghammer and Van Den Berge, 2019). It seems to be a good explanation for the current findings of PD pathology, but also makes the study of the role of intestinal dysbiosis in PD more difficult. But at the same time, it provides a way of thinking, that is, by dividing the test subjects into RBD positive group and $\mathrm{RBD}$ negative group, will there be different findings?

Thus far, microbial therapies including probiotics, prebiotics, and FMT have been shown to improve constipation, which is the most important GI symptom reported by PD patients. Microbial therapy is expected to represent a novel method for the treatment of constipation in PD patients, and large-sample clinical trials are necessary to further confirm the efficacy and safety of these treatments before their formal application.

\section{AUTHOR CONTRIBUTIONS}

YH wrote the first draft. JL co-wrote the first draft. XL and YZ made substantial contribution to the content. XC and LL conceptualized the structure and edited the final version of the manuscript. All authors contributed to the article and approved the submitted version.

\section{FUNDING}

This work is supported by grants from the Natural Science Foundation of China (81501117), the Natural Science Foundation of Guangdong Province (2018A0303130307), Guangdong Basic and Applied Basic Research Foundation (2019A1515011739), and the Fundamental Research Funds for the Central Universities (19ykpy27). 


\section{REFERENCES}

Adak, A., and Khan, M. R. (2019). An Insight Into Gut Microbiota and its Functionalities. Cell. Mol. Life Sci. CMLS 76, 473-493. doi: 10.1007/s00018-018-2943-4

Aho, V. T. E., Pereira, P. A. B., Voutilainen, S., Paulin, L., Pekkonen, E., Auvinen, P., et al. (2019). Gut Microbiota in Parkinson's Disease: Temporal Stability and Relations to Disease Progression. EBioMedicine 44, 691-707. doi: 10.1016/j.ebiom.2019.05.064

Andrews, Z. B., Erion, D., Beiler, R., Liu, Z. W., Abizaid, A., Zigman, J., et al. (2009). Ghrelin Promotes and Protects Nigrostriatal Dopamine Function Via a UCP2-Dependent Mitochondrial Mechanism. J. Neurosci. 29, 14057-14065. doi: 10.1523/jneurosci.3890-09.2009

Baizabal-Carvallo, J. F., and Alonso-Juarez, M. (2020). The Link Between Gut Dysbiosis and Neuroinflammation in Parkinson's Disease. Neuroscience 432, 160-173. doi: 10.1016/j.neuroscience.2020.02.030

Baldini, F., Hertel, J., Sandt, E., Thinnes, C. C., Neuberger-Castillo, L., Pavelka, L., et al. (2020). Parkinson's Disease-Associated Alterations of the Gut Microbiome Predict Disease-Relevant Changes in Metabolic Functions. BMC Biol. 18, 62. doi: 10.1186/s12915-020-00775-7

Barichella, M., Pacchetti, C., Bolliri, C., Cassani, E., Iorio, L., Pusani, C., et al. (2016). Probiotics and Prebiotic Fiber for Constipation Associated With Parkinson Disease: An RCT. Neurology 87, 1274-1280. doi: 10.1212/ wnl.0000000000003127

Bedarf, J. R., Hildebrand, F., Coelho, L. P., Sunagawa, S., Bahram, M., Goeser, F., et al. (2017). Functional Implications of Microbial and Viral Gut Metagenome Changes in Early Stage L-DOPA-Naive Parkinson's Disease Patients. Genome Med. 9, 39. doi: 10.1186/s13073-017-0428-y

Beier, E. E., Neal, M., Alam, G., Edler, M., Wu, L. J., and Richardson, J. R. (2017). Alternative Microglial Activation is Associated With Cessation of Progressive Dopamine Neuron Loss in Mice Systemically Administered Lipopolysaccharide. Neurobiol. Dis. 108, 115-127. doi: 10.1016/j.nbd.2017.08.009

Blanco, L. P., Evans, M. L., Smith, D. R., Badtke, M. P., and Chapman, M. R. (2012). Diversity, Biogenesis and Function of Microbial Amyloids. Trends Microbiol. 20, 66-73. doi: 10.1016/j.tim.2011.11.005

Borghammer, P., and Van Den Berge, N. (2019). Brain-First Versus Gut-First Parkinson's Disease: A Hypothesis. J. Parkinsons Dis. 9, S281-S295. doi: 10.3233/jpd-191721

Braak, H., Rub, U., Gai, W. P., and Del Tredici, K. (2003). Idiopathic Parkinson's Disease: Possible Routes by Which Vulnerable Neuronal Types may be Subject to Neuroinvasion by an Unknown Pathogen. J. Neural Transm. (Vienna Austria: 1996) 110, 517-536. doi: 10.1007/s00702-002-0808-2

Braniste, V., Al-Asmakh, M., Kowal, C., Anuar, F., Abbaspour, A., Toth, M., et al. (2014). The Gut Microbiota Influences Blood-Brain Barrier Permeability in Mice. Sci. Trans. Med. 6, 263ra158. doi: 10.1126/scitranslmed.3009759

Caggiu, E., Paulus, K., Arru, G., Piredda, R., Sechi, G. P., and Sechi, L. A. (2016). Humoral Cross Reactivity Between $\alpha$-Synuclein and Herpes Simplex-1 Epitope in Parkinson's Disease, a Triggering Role in the Disease? J. Neuroimmunol. 291, 110-114. doi: 10.1016/j.jneuroim.2016.01.007

Cahenzli, J., Balmer, M. L., and McCoy, K. D. (2013). Microbial-Immune CrossTalk and Regulation of the Immune System. Immunology 138, 12-22. doi: $10.1111 / j .1365-2567.2012 .03624 . x$

Caputi, V., and Giron, M. (2018). Microbiome-Gut-Brain Axis and Toll-Like Receptors in Parkinson's Disease. Int. J. Mol. Sci. 19, 1689. doi: 10.3390/ ijms 19061689

Cassani, E., Privitera, G., Pezzoli, G., Pusani, C., Madio, C., Iorio, L., et al. (2011). Use of Probiotics for the Treatment of Constipation in Parkinson's Disease Patients. Minerva Gastroenterol. Dietol. 57, 117-121.

Cersosimo, M. G., Raina, G. B., Pecci, C., Pellene, A., Calandra, C. R., Gutierrez, C., et al. (2013). Gastrointestinal Manifestations in Parkinson's Disease: Prevalence and Occurrence Before Motor Symptoms. J. Neurol. 260, 13321338. doi: $10.1007 / \mathrm{s} 00415-012-6801-2$

Chang, P. V., Hao, L., Offermanns, S., and Medzhitov, R. (2014). The Microbial Metabolite Butyrate Regulates Intestinal Macrophage Function Via Histone Deacetylase Inhibition. Proc. Natl. Acad. Sci. U. S. A. 111, 2247-2252. doi: 10.1073/pnas.1322269111

Chen, S. G., Stribinskis, V., Rane, M. J., Demuth, D. R., Gozal, E., Roberts, A. M., et al. (2016). Exposure to the Functional Bacterial Amyloid Protein Curli Enhances Alpha-Synuclein Aggregation in Aged Fischer 344 Rats and Caenorhabditis Elegans. Sci. Rep. 6, 34477. doi: 10.1038/srep34477
Cho, C. E., Taesuwan, S., Malysheva, O. V., Bender, E., Tulchinsky, N. F., Yan, J., et al. (2017). Trimethylamine-N-oxide (TMAO) response to animal source foods varies among healthy young men and is influenced by their gut microbiota composition: A randomized controlled trial. Mol. Nut. Food Res. 61, 1600324. doi: 10.1002/mnfr.201600324

Choi, J. G., Kim, N., Ju, I. G., Eo, H., Lim, S. M., Jang, S. E., et al. (2018). Oral Administration of Proteus Mirabilis Damages Dopaminergic Neurons and Motor Functions in Mice. Sci. Rep. 8, 1275. doi: 10.1038/s41598-018-19646-x

Choi, D. Y., Zhang, J., and Bing, G. (2010). Aging Enhances the Neuroinflammatory Response and Alpha-Synuclein Nitration in Rats. Neurobiol. Aging 31, 1649-1653. doi: 10.1016/j.neurobiolaging.2008.09.010

Chorell, E., Andersson, E., Evans, M. L., Jain, N., Götheson, A., Åden, J., et al. (2015). Bacterial Chaperones Csge and Csge Differentially Modulate Human $\alpha$-Synuclein Amyloid Formation Via Transient Contacts. PloS One 10, e0140194. doi: 10.1371/journal.pone.0140194

Collins, S. M., Surette, M., and Bercik, P. (2012). The Interplay Between the Intestinal Microbiota and the Brain. Nat. Rev. Microbiol. 10, 735-742. doi: $10.1038 /$ nrmicro2876

Cryan, J. F., O’Riordan, K. J., Cowan, C. S. M., Sandhu, K. V., Bastiaanssen, T. F. S., Boehme, M., et al. (2019). The Microbiota-Gut-Brain Axis. Physiol. Rev. 99, 1877-2013. doi: 10.1152/physrev.00018.2018

Cummings, J. H., Pomare, E. W., Branch, W. J., Naylor, C. P., and Macfarlane, G. T. (1987). Short Chain Fatty Acids in Human Large Intestine, Portal, Hepatic and Venous Blood. Gut 28, 1221-1227. doi: 10.1136/gut.28.10.1221

Del Tredici, K., and Braak, H. (2008). A Not Entirely Benign Procedure: Progression of Parkinson's Disease. Acta Neuropathol. 115, 379-384. doi: 10.1007/s00401-008-0355-5

De Vadder, F., Kovatcheva-Datchary, P., Goncalves, D., Vinera, J., Zitoun, C., Duchampt, A., et al. (2014). Microbiota-Generated Metabolites Promote Metabolic Benefits Via Gut-Brain Neural Circuits. Cell 156, 84-96. doi: 10.1016/j.cell.2013.12.016

Devos, D., Lebouvier, T., Lardeux, B., Biraud, M., Rouaud, T., Pouclet, H., et al. (2013). Colonic Inflammation in Parkinson's Disease. Neurobiol. Dis. 50, 4248. doi: 10.1016/j.nbd.2012.09.007

Dorsey, E. R., Constantinescu, R., Thompson, J. P., Biglan, K. M., Holloway, R. G., Kieburtz, K., et al. (2007). Projected Number of People With Parkinson Disease in the Most Populous NationThrough 2030. Neurology 68, 384-386. doi: 10.1212/01.wnl.0000247740.47667.03

Eisenhofer, G., Aneman, A., Friberg, P., Hooper, D., Fåndriks, L., Lonroth, H., et al. (1997). Substantial Production of Dopamine in the Human Gastrointestinal Tract. J. Clin. Endocrinol. Metab. 82, 3864-3871. doi: $10.1210 /$ jcem.82.11.4339

Erny, D., Hrabe de Angelis, A. L., Jaitin, D., Wieghofer, P., Staszewski, O., David, E., et al. (2015). Host Microbiota Constantly Control Maturation and Function of Microglia in the CNS. Nat. Neurosci. 18, 965-977. doi: 10.1038/nn.4030

Fitzgerald, E., Murphy, S., and Martinson, H. A. (2019). Alpha-Synuclein Pathology and the Role of the Microbiota in Parkinson's Disease. Front. Neurosci. 13, 369. doi: 10.3389/fnins.2019.00369

Flores-Martinez, Y. M., Fernandez-Parrilla, M. A., Ayala-Davila, J., Reyes-Corona, D., Blanco-Alvarez, V. M., Soto-Rojas, L. O., et al. (2018). Acute Neuroinflammatory Response in the Substantia Nigra Pars Compacta of Rats After a Local Injection of Lipopolysaccharide. J. Immunol. Res. 2018, 1838921. doi: 10.1155/2018/1838921

Forsyth, C. B., Shannon, K. M., Kordower, J. H., Voigt, R. M., Shaikh, M., Jaglin, J. A., et al. (2011). Increased Intestinal Permeability Correlates With Sigmoid Mucosa Alpha-Synuclein Staining and Endotoxin Exposure Markers in Early Parkinson's Disease. PloS One 6, e28032. doi: 10.1371/journal.pone.0028032

Gao, K., Pi, Y., Mu, C. L., Peng, Y., Huang, Z., and Zhu, W. Y. (2018). AntibioticsInduced Modulation of Large Intestinal Microbiota Altered Aromatic Amino Acid Profile and Expression of Neurotransmitters in the Hypothalamus of Piglets. J. Neurochem. 146, 219-234. doi: 10.1111/jnc.14333

Georgescu, D., Ancusa, O. E., Georgescu, L. A., Ionita, I., and Reisz, D. (2016). Nonmotor Gastrointestinal Disorders in Older Patients With Parkinson's Disease: Is There Hope? Clin. Interv. Aging 11, 1601-1608. doi: 10.2147/ cia.S106284

Gerhardt, S., and Mohajeri, M. H. (2018). Changes of Colonic Bacterial Composition in Parkinson's Disease and Other Neurodegenerative Diseases. Nutrients 10, 708. doi: 10.3390/nu10060708 
Ghosh, T. S., Gupta, S. S., Bhattacharya, T., Yadav, D., Barik, A., Chowdhury, A., et al. (2014). Gut microbiomes of Indian children of varying nutritional status. PLoS One 9, e95547. doi: 10.1371/journal.pone.0095547

Gibson, G. R., Hutkins, R., Sanders, M. E., Prescott, S. L., Reimer, R. A., Salminen, S. J., et al. (2017). Expert Consensus Document: The International Scientific Association for Probiotics and Prebiotics (ISAPP) Consensus Statement on the Definition and Scope of Prebiotics. Nat. Rev. Gastroenterol. Hepatol. 14, 491502. doi: 10.1038/nrgastro.2017.75

Gioacchini, G., Ciani, E., Pessina, A., Cecchini, C., Silvi, S., Rodiles, A., et al. (2018). Effects of Lactogen 13, a New Probiotic Preparation, on Gut Microbiota and Endocrine Signals Controlling Growth and Appetite of Oreochromis Niloticus Juveniles. Microb. Ecol. 76, 1063-1074. doi: 10.1007/s00248-018-1177-1

Giongo, A., Gano, K. A., Crabb, D. B., Mukherjee, N., Novelo, L. L., Casella, G., et al. (2011). Toward defining the autoimmune microbiome for type 1 diabetes. The ISME journal 5, 82-91. doi: 10.1038/ismej.2010.92

Goya, M. E., Xue, F., Sampedro-Torres-Quevedo, C., Arnaouteli, S., RiquelmeDominguez, L., Romanowski, A., et al. (2020). Probiotic Bacillus Subtilis Protects Against $\alpha$-Synuclein Aggregation in C. Elegans. Cell Rep. 30, 367380.e367. doi: 10.1016/j.celrep.2019.12.078

Gutsmann, T., Müller, M., Carroll, S. F., MacKenzie, R. C., Wiese, A., and Seydel, U. (2001). Dual Role of Lipopolysaccharide (LPS)-Binding Protein in Neutralization of LPS and Enhancement of LPS-Induced Activation of Mononuclear Cells. Infect. Immun. 69, 6942-6950. doi: 10.1128/ iai.69.11.6942-6950.2001

Hasegawa, S., Goto, S., Tsuji, H., Okuno, T., Asahara, T., Nomoto, K., et al. (2015). Intestinal Dysbiosis and Lowered Serum Lipopolysaccharide-Binding Protein in Parkinson's Disease. PloS One 10, e0142164. doi: 10.1371/ journal.pone. 0142164

Hashim, H., Azmin, S., Razlan, H., Yahya, N. W., Tan, H. J., Manaf, M. R., et al. (2014). Eradication of Helicobacter Pylori Infection Improves Levodopa Action, Clinical Symptoms and Quality of Life in Patients With Parkinson's Disease. PloS One 9, e112330. doi: 10.1371/journal.pone.0112330

Helander, H. F., and Fandriks, L. (2014). Surface Area of the Digestive Tract Revisited. Scand. J. Gastroenterol. 49, 681-689. doi: 10.3109/ 00365521.2014 .898326

Hill-Burns, E. M., Debelius, J. W., Morton, J. T., Wissemann, W. T., Lewis, M. R., Wallen, Z. D., et al. (2017). Parkinson's Disease and Parkinson's Disease Medications Have Distinct Signatures of the Gut Microbiome. Movement Disord. 32, 739-749. doi: 10.1002/mds.26942

Hill, C., Guarner, F., Reid, G., Gibson, G. R., Merenstein, D. J., Pot, B., et al. (2014). Expert Consensus Document. The International Scientific Association for Probiotics and Prebiotics Consensus Statement on the Scope and Appropriate Use of the Term Probiotic. Nat. Rev. Gastroenterol. Hepatol. 11, 506-514. doi: 10.1038/nrgastro.2014.66

Hirsch, E. C., and Hunot, S. (2009). Neuroinflammation in Parkinson's Disease: A Target for Neuroprotection? Lancet Neurol. 8, 382-397. doi: 10.1016/s14744422(09)70062-6

Hoban, D. B., Connaughton, E., Connaughton, C., Hogan, G., Thornton, C., Mulcahy, P., et al. (2013). Further Characterisation of the LPS Model of Parkinson's Disease: A Comparison of Intra-Nigral and Intra-Striatal Lipopolysaccharide Administration on Motor Function, Microgliosis and Nigrostriatal Neurodegeneration in the Rat. Brain Behav. Immun. 27, 91100. doi: 10.1016/j.bbi.2012.10.001

Hopfner, F., Künstner, A., Müller, S. H., Künzel, S., Zeuner, K. E., Margraf, N. G., et al. (2017). Gut Microbiota in Parkinson Disease in a Northern German Cohort. Brain Res. 1667, 41-45. doi: 10.1016/j.brainres.2017.04.019

Huang, H., Xu, H., Luo, Q., He, J., Li, M., Chen, H., et al. (2019). Fecal Microbiota Transplantation to Treat Parkinson's Disease With Constipation: A Case Report. Medicine 98, e16163. doi: 10.1097/md.0000000000016163

Hunter, R. L., Cheng, B., Choi, D. Y., Liu, M., Liu, S., Cass, W. A., et al. (2009). Intrastriatal Lipopolysaccharide Injection Induces Parkinsonism in C57/B6 Mice. J. Neurosci. Res. 87, 1913-1921. doi: 10.1002/jnr.22012

Jin, M., Li, J., Liu, F., Lyu, N., Wang, K., Wang, L., et al. (2019). Analysis of the Gut Microflora in Patients With Parkinson's Disease. Front. Neurosci. 13, 1184. doi: 10.3389/fnins.2019.01184

Just, S., Mondot, S., Ecker, J., Wegner, K., Rath, E., Gau, L., et al. (2018). The gut microbiota drives the impact of bile acids and fat source in diet on mouse metabolism. Microbiome 6, 134. doi: 10.1186/s40168-018-0510-8
Kalia, L. V., and Lang, A. E. (2015). Parkinson's Disease. Lancet (London England) 386, 896-912. doi: 10.1016/s0140-6736(14)61393-3

Karlsson, F. H., Tremaroli, V., Nookaew, I., Bergstrom, G., Behre, C. J., Fagerberg, B., et al. (2013). Gut metagenome in European women with normal, impaired and diabetic glucose control. Nature 498, 99-103. doi: 10.1038/nature12198

Kawasaki, T., and Kawai, T. (2014). Toll-Like Receptor Signaling Pathways. Front. Immunol. 5, 461. doi: 10.3389/fimmu.2014.00461

Keshavarzian, A., Green, S. J., Engen, P. A., Voigt, R. M., Naqib, A., Forsyth, C. B., et al. (2015). Colonic Bacterial Composition in Parkinson's Disease. Movement Disord. 30, 1351-1360. doi: 10.1002/mds.26307

Kim, M. H., Kang, S. G., Park, J. H., Yanagisawa, M., and Kim, C. H. (2013). ShortChain Fatty Acids Activate GPR41 and GPR43 on Intestinal Epithelial Cells to Promote Inflammatory Responses in Mice. Gastroenterology 145, 396406.e391-310. doi: 10.1053/j.gastro.2013.04.056

Kim, S., Kwon, S. H., Kam, T. I., Panicker, N., Karuppagounder, S. S., Lee, S., et al. (2019). Transneuronal Propagation of Pathologic $\alpha$-Synuclein From the Gut to the Brain Models Parkinson's Disease. Neuron 103, 627-641.e627. doi: 10.1016/j.neuron.2019.05.035

Klein, R. D., Shu, Q., Cusumano, Z. T., Nagamatsu, K., Gualberto, N. C., Lynch, A. J. L., et al. (2018). Structure-Function Analysis of the Curli Accessory Protein Csge Defines Surfaces Essential for Coordinating Amyloid Fiber Formation. mBio 9, e01349-18. doi: 10.1128/mBio.01349-18

Koh, A., De Vadder, F., Kovatcheva-Datchary, P., and Backhed, F. (2016). From Dietary Fiber to Host Physiology: Short-Chain Fatty Acids as Key Bacterial Metabolites. Cell 165, 1332-1345. doi: 10.1016/j.cell.2016.05.041

Lin, C. H., Chen, C. C., Chiang, H. L., Liou, J. M., Chang, C. M., Lu, T. P., et al. (2019). Altered Gut Microbiota and Inflammatory Cytokine Responses in Patients With Parkinson's Disease. J. Neuroinflamm. 16, 129. doi: 10.1186/ s12974-019-1528-y

Lin, A., Zheng, W., He, Y., Tang, W., Wei, X., He, R., et al. (2018). Gut Microbiota in Patients With Parkinson's Disease in Southern China. Parkinsonism Relat. Disord. 53, 82-88. doi: 10.1016/j.parkreldis.2018.05.007

Liu, M., and Bing, G. (2011). Lipopolysaccharide Animal Models for Parkinson's Disease. Parkinson's Dis. 2011, 327089. doi: 10.4061/2011/327089

Liu, B., Fang, F., Pedersen, N. L., Tillander, A., Ludvigsson, J. F., Ekbom, A., et al. (2017). Vagotomy and Parkinson Disease: A Swedish Register-Based MatchedCohort Study. Neurology 88, 1996-2002. doi: 10.1212/wnl.0000000000003961

Li, W., Wu, X., Hu, X., Wang, T., Liang, S., Duan, Y., et al. (2017). Structural Changes of Gut Microbiota in Parkinson's Disease and its Correlation With Clinical Features. Sci. Chin. Life Sci. 60, 1223-1233. doi: 10.1007/s11427-0169001-4

Lozupone, C. A., Stombaugh, J. I., Gordon, J. I., Jansson, J. K., and Knight, R. (2012). Diversity, Stability and Resilience of the Human Gut Microbiota. Nature 489, 220-230. doi: 10.1038/nature11550

Magistrelli, L., Amoruso, A., Mogna, L., Graziano, T., Cantello, R., Pane, M., et al. (2019). Probiotics may Have Beneficial Effects in Parkinson's Disease: in Vitro Evidence. Front. Immunol. 10, 969. doi: 10.3389/fimmu.2019.00969

Mattam, U., and Jagota, A. (2015). Daily Rhythms of Serotonin Metabolism and the Expression of Clock Genes in Suprachiasmatic Nucleus of RotenoneInduced Parkinson's Disease Male Wistar Rat Model and Effect of Melatonin Administration. Biogerontology 16, 109-123. doi: 10.1007/s10522-014-9541-0

McGee, D. J., Lu, X. H., and Disbrow, E. A. (2018). Stomaching the Possibility of a Pathogenic Role for Helicobacter Pylori in Parkinson's Disease. J. Parkinsons Dis. 8, 367-374. doi: 10.3233/JPD-181327

Meksawan, K., Chaotrakul, C., Leeaphorn, N., Gonlchanvit, S., Eiam-Ong, S., and Kanjanabuch, T. (2016). Effects of Fructo-Oligosaccharide Supplementation on Constipation in Elderly Continuous Ambulatory Peritoneal Dialysis Patients. Peritoneal Dialysis Int. 36, 60-66. doi: 10.3747/pdi.2014.00015

Miraglia, F., and Colla, E. (2019). Microbiome, Parkinson's Disease and Molecular Mimicry. Cells 8, 222. doi: 10.3390/cells8030222

Mohle, L., Mattei, D., Heimesaat, M. M., Bereswill, S., Fischer, A., Alutis, M., et al. (2016). Ly6C(Hi) Monocytes Provide a Link Between Antibiotic-Induced Changes in Gut Microbiota and Adult Hippocampal Neurogenesis. Cell Rep. 15, 1945-1956. doi: 10.1016/j.celrep.2016.04.074

Mukherjee, A., Biswas, A., and Das, S. K. (2016). Gut Dysfunction in Parkinson's Disease. World J. Gastroenterol. 22, 5742-5752. doi: 10.3748/wjg.v22.i25.5742

Murakami, M., Ohta, T., and Ito, S. (2009). Lipopolysaccharides Enhance the Action of Bradykinin in Enteric Neurons Via Secretion of Interleukin-1beta 
From Enteric Glial Cells. J. Neurosci. Res. 87, 2095-2104. doi: 10.1002/ jnr.22036

Nair, A. T., Ramachandran, V., Joghee, N. M., Antony, S., and Ramalingam, G. (2018). Gut Microbiota Dysfunction as Reliable non-Invasive Early Diagnostic Biomarkers in the Pathophysiology of Parkinson's Disease: A Critical Review. J. Neurogastroenterol. Motil. 24, 30-42. doi: 10.5056/jnm17105

Ozansoy, M., and Başak, A. N. (2013). The Central Theme of Parkinson's Disease: Q-Synuclein. Mol. Neurobiol. 47, 460-465. doi: 10.1007/s12035-012-8369-3

Pal, G. D., Shaikh, M., Forsyth, C. B., Ouyang, B., Keshavarzian, A., and Shannon, K. M. (2015). Abnormal Lipopolysaccharide Binding Protein as Marker of Gastrointestinal Inflammation in Parkinson Disease. Front. Neurosci. 9, 306. doi: $10.3389 /$ fnins.2015.00306

Parracho, H. M., Bingham, M. O., Gibson, G. R., and McCartney, M. L. (2005). Differences between the gut microflora of children with autistic spectrum disorders and that of healthy children. J. Med. Microbiol. 54, 987-991. doi: 10.1099/jmm.0.46101-0

Pellegrini, C., Antonioli, L., Calderone, V., Colucci, R., Fornai, M., and Blandizzi, C. (2020). Microbiota-Gut-Brain Axis in Health and Disease: Is NLRP3 Inflammasome At the Crossroads of Microbiota-Gut-Brain Communications? Prog. Neurobiol. 191, 101806. doi: 10.1016/j.pneurobio.2020.101806

Peralta Ramos, J. M., Iribarren, P., Bousset, L., Melki, R., Baekelandt, V., and Van der Perren, A. (2019). Peripheral Inflammation Regulates CNS Immune Surveillance Through the Recruitment of Inflammatory Monocytes Upon Systemic Alpha-Synuclein Administration. Front. Immunol. 10, 80. doi: $10.3389 /$ fimmu.2019.00080

Perez-Pardo, P., de Jong, E. M., Broersen, L. M., van Wijk, N., Attali, A., Garssen, J., et al. (2017). Promising Effects of Neurorestorative Diets on Motor, Cognitive, and Gastrointestinal Dysfunction After Symptom Development in a Mouse Model of Parkinson's Disease. Front. Aging Neurosci. 9, 57. doi: 10.3389/fnagi.2017.00057

Perez-Pardo, P., Dodiya, H. B., Engen, P. A., Forsyth, C. B., Huschens, A. M., Shaikh, M., et al. (2019). Role of TLR4 in the Gut-Brain Axis in Parkinson's Disease: A Translational Study From Men to Mice. Gut 68, 829-843. doi: 10.1136/gutjnl-2018-316844

Perez-Pardo, P., Dodiya, H. B., Engen, P. A., Naqib, A., Forsyth, C. B., Green, S. J., et al. (2018). Gut Bacterial Composition in a Mouse Model of Parkinson's Disease. Benef. Microbes 9, 799-814. doi: 10.3920/bm2017.0202

Petrov, V. A., Saltykova, I. V., Zhukova, I. A., Alifirova, V. M., Zhukova, N. G., Dorofeeva, Y. B., et al. (2017). Analysis of Gut Microbiota in Patients With Parkinson's Disease. Bull. Exp. Biol. Med. 162, 734-737. doi: 10.1007/s10517-017-3700-7

Ploger, S., Stumpff, F., Penner, G. B., Schulzke, J. D., Gabel, G., Martens, H., et al. (2012). Microbial Butyrate and its Role for Barrier Function in the Gastrointestinal Tract. Ann. New York Acad. Sci. 1258, 52-59. doi: 10.1111/ j.1749-6632.2012.06553.x

Punsoni, M., Friedman, J. H., Resnick, M., Donahue, J. E., Yang, D. F., and Stopa, E. G. (2019). Enteric Pathologic Manifestations of Alpha-Synucleinopathies. Appl. Immunohistochem. Mol. Morphol. 27, 543-548. doi: 10.1097/ pai.0000000000000613

Qian, Y., Yang, X., Xu, S., Wu, C., Song, Y., Qin, N., et al. (2018). Alteration of the Fecal Microbiota in Chinese Patients With Parkinson's Disease. Brain Behav. Immun. 70, 194-202. doi: 10.1016/j.bbi.2018.02.016

Rooks, M. G., and Garrett, W. S. (2016). Gut Microbiota, Metabolites and Host Immunity. Nat. Rev. Immunol. 16, 341-352. doi: 10.1038/nri.2016.42

Round, J. L., and Mazmanian, S. K. (2009). The Gut Microbiota Shapes Intestinal Immune Responses During Health and Disease. Nat. Rev. Immunol. 9, 313323. doi: $10.1038 /$ nri2515

Sampson, T. R., Debelius, J. W., Thron, T., Janssen, S., Shastri, G. G., Ilhan, Z. E., et al. (2016). Gut Microbiota Regulate Motor Deficits and Neuroinflammation in a Model of Parkinson's Disease. Cell 167, 1469-1480.e1412. doi: 10.1016/ j.cell.2016.11.018

Scheperjans, F., Aho, V., Pereira, P. A., Koskinen, K., Paulin, L., Pekkonen, E., et al. (2015). Gut Microbiota are Related to Parkinson's Disease and Clinical Phenotype. Movement Disord. 30, 350-358. doi: 10.1002/mds.26069

Sender, R., Fuchs, S., and Milo, R. (2016a). Are We Really Vastly Outnumbered? Revisiting the Ratio of Bacterial to Host Cells in Humans. Cell 164, 337-340. doi: 10.1016/j.cell.2016.01.013

Sender, R., Fuchs, S., and Milo, R. (2016b). Revised Estimates for the Number of Human and Bacteria Cells in the Body. PloS Biol. 14, e1002533. doi: 10.1371/ journal.pbio. 1002533
Sergeyeva, T. N., and Sergeyev, V. G. (2011). Administration of LPS-Stimulated Autologous Macrophages Induces Alpha-Synuclein Aggregation in Dopaminergic Neurons of Rat Brain. Bull. Exp. Biol. Med. 150, 406-408. doi: 10.1007/s10517-011-1153-y

Shannon, K. M., Keshavarzian, A., Dodiya, H. B., Jakate, S., and Kordower, J. H. (2012). Is Alpha-Synuclein in the Colon a Biomarker for Premotor Parkinson's Disease? Evidence From 3 Cases. Movement Disord. 27, 716-719. doi: 10.1002/ mds. 25020

Sharon, G., Sampson, T. R., Geschwind, D. H., and Mazmanian, S. K. (2016). The Central Nervous System and the Gut Microbiome. Cell 167, 915-932. doi: 10.1016/j.cell.2016.10.027

Shen, X., Yang, H., Wu, Y., Zhang, D., and Jiang, H. (2017). Meta-Analysis: Association of Helicobacter Pylori Infection With Parkinson's Diseases. Helicobacter 22, e12398. doi: 10.1111/hel.12398

Sochocka, M., Donskow-Lysoniewska, K., Diniz, B. S., Kurpas, D., Brzozowska, E., Leszek, J., et al. (2019). The Gut Microbiome Alterations and InflammationDriven Pathogenesis of Alzheimer's Disease-a Critical Review. Mol. Neurobiol. 56, 1841-1851. doi: 10.1007/s12035-018-1188-4

Soret, R., Chevalier, J., De Coppet, P., Poupeau, G., Derkinderen, P., Segain, J. P., et al. (2010). Short-Chain Fatty Acids Regulate the Enteric Neurons and Control Gastrointestinal Motility in Rats. Gastroenterology 138, 1772-1782. doi: 10.1053/j.gastro.2010.01.053

Sudo, N., Chida, Y., Aiba, Y., Sonoda, J., Oyama, N., Yu, X. N., et al. (2004). Postnatal Microbial Colonization Programs the Hypothalamic-PituitaryAdrenal System for Stress Response in Mice. J. Physiol. 558, 263-275. doi: $10.1113 /$ jphysiol.2004.063388

Sun, M. F., Zhu, Y. L., Zhou, Z. L., Jia, X. B., Xu, Y. D., Yang, Q., et al. (2018). Neuroprotective Effects of Fecal Microbiota Transplantation on MPTPInduced Parkinson's Disease Mice: Gut Microbiota, Glial Reaction and TLR4/TNF-Alpha Signaling Pathway. Brain Behav. Immun. 70, 48-60. doi: 10.1016/j.bbi.2018.02.005

Svensson, E., Horvath-Puho, E., Thomsen, R. W., Djurhuus, J. C., Pedersen, L., Borghammer, P., et al. (2015). Vagotomy and Subsequent Risk of Parkinson's Disease. Ann. Neurol. 78, 522-529. doi: 10.1002/ana.24448

Tamtaji, O. R., Taghizadeh, M., Daneshvar Kakhaki, R., Kouchaki, E., Bahmani, F., Borzabadi, S., et al. (2019). Clinical and Metabolic Response to Probiotic Administration in People With Parkinson's Disease: A Randomized, DoubleBlind, Placebo-Controlled Trial. Clin. Nutr. 38, 1031-1035. doi: 10.1016/ j.clnu.2018.05.018

Tan, A. H., Lim, S. Y., Chong, K. K., MAA, A. M., Hor, J. W., Lim, J. L., et al. (2021). Probiotics for Constipation in Parkinson Disease: A Randomized Placebo-Controlled Study. Neurology 96, e772-e782. doi: 10.1212/ wnl.0000000000010998

Tan, A. H., Lim, S. Y., Mahadeva, S., Loke, M. F., Tan, J. Y., Ang, B. H., et al. (2020). Helicobacter Pylori Eradication in Parkinson's Disease: A Randomized Placebo-Controlled Trial. Movement Disord. 35, 2250-2260. doi: 10.1002/ mds. 28248

Torres-Fuentes, C., Golubeva, A. V., Zhdanov, A. V., Wallace, S., Arboleya, S., Papkovsky, D. B., et al. (2019). Short-Chain Fatty Acids and Microbiota Metabolites Attenuate Ghrelin Receptor Signaling. FASEB J. 33, 1354613559. doi: 10.1096/fj.201901433R

Turnbaugh, P J., Backhed, F., Fulton, L., Gordon, J. I., et al. (2008). Diet-induced obesity is linked to marked but reversible alterations in the mouse distal gut microbiome. Cell Host \& Microbe 3, 213-223. doi: 10.1016/j.chom.2008.02.015

Tursi, S. A., and Tükel, Ç. (2018). Curli-Containing Enteric Biofilms Inside and Out: Matrix Composition, Immune Recognition, and Disease Implications. Microbiol. Mol. Biol. Rev. 82, e00028-18. doi: 10.1128/mmbr.00028-18

Unger, M. M., Spiegel, J., Dillmann, K. U., Grundmann, D., Philippeit, H., Burmann, J., et al. (2016). Short Chain Fatty Acids and Gut Microbiota Differ Between Patients With Parkinson's Disease and Age-Matched Controls. Parkinsonism Relat. Disord. 32, 66-72. doi: 10.1016/j.parkreldis.2016.08.019

Van Den Berge, N., Ferreira, N., Gram, H., Mikkelsen, T. W., Alstrup, A. K. O., Casadei, N., et al. (2019). Evidence for Bidirectional and Trans-Synaptic Parasympathetic and Sympathetic Propagation of Alpha-Synuclein in Rats. Acta Neuropathol. 138, 535-550. doi: 10.1007/s00401-019-02040-w

van, I. S. C. D., and Derkinderen, P. (2019). The Intestinal Barrier in Parkinson's Disease: Current State of Knowledge. J. Parkinsons Dis. 9, S323-S329. doi: $10.3233 /$ jpd-191707 
Verbaan, D., Marinus, J., Visser, M., van Rooden, S. M., Stiggelbout, A. M., and van Hilten, J. J. (2007). Patient-Reported Autonomic Symptoms in Parkinson Disease. Neurology 69, 333-341. doi: 10.1212/01.wnl.0000266593.50534.e8

Vidal-Martinez, G., Chin, B., Camarillo, C., Herrera, G. V., Yang, B., Sarosiek, I., et al. (2020). A Pilot Microbiota Study in Parkinson's Disease Patients Versus Control Subjects, and Effects of FTY720 and FTY720-Mitoxy Therapies in Parkinsonian and Multiple System Atrophy Mouse Models. J. Parkinsons Dis. 10, 185-192. doi: 10.3233/JPD-191693

Vogt, N. M., Dill-McFarland, K. A., Harding, S. J., Merluzzi, A. P., Johnson, S. C., Carlsson, C. M., et al (2017). Bacterial Neuroactive Compounds Produced by Psychobiotics. Gut microbiome alterations in Alzheimer's disease. Sci Rep 7, 13537. doi: 10.1038/s41598-017-13601-y

Wall, R., Cryan, J. F., Ross, R. P., Fitzgerald, G. F., Dinan, T. G., and Stanton, C. (2014). Bacterial Neuroactive Compounds Produced by Psychobiotics. Adv. Exp. Med. Biol. 817, 221-239. doi: 10.1007/978-1-4939-0897-4_10

Wang, H. B., Wang, P. Y., Wang, X., Wan, Y. L., and Liu, Y. C. (2012). Butyrate Enhances Intestinal Epithelial Barrier Function Via Up-Regulation of Tight Junction Protein Claudin-1 Transcription. Dig. Dis. Sci. 57, 3126-3135. doi: 10.1007/s10620-012-2259-4

Weng, R., Shen, S., Burton, C., Yang, L., Nie, H., Tian, Y., et al. (2016). Lipidomic Profiling of Tryptophan Hydroxylase 2 Knockout Mice Reveals Novel Lipid Biomarkers Associated With Serotonin Deficiency. Anal. Bioanal. Chem. 408, 2963-2973. doi: 10.1007/s00216-015-9256-3
Woulfe, J. M., Gray, M. T., Gray, D. A., Munoz, D. G., and Middeldorp, J. M (2014). Hypothesis: A Role for EBV-Induced Molecular Mimicry in Parkinson's Disease. Parkinsonism Relat. Disord. 20, 685-694. doi: 10.1016/ j.parkreldis.2014.02.031

Yang, X., Qian, Y., Xu, S., Song, Y., and Xiao, Q. (2017). Longitudinal Analysis of Fecal Microbiome and Pathologic Processes in a Rotenone Induced Mice Model of Parkinson's Disease. Front. Aging Neurosci. 9, 441. doi: 10.3389/ fnagi.2017.00441

Zendehdel, A., and Roham, M. (2020). Role of Helicobacter Pylori Infection in the Manifestation of Old Age-Related Diseases. Mol. Genet. Genomic. Med. 8, e1157. doi: $10.1002 / \mathrm{mgg} 3.1157$

Conflict of Interest: The authors declare that the research was conducted in the absence of any commercial or financial relationships that could be construed as a potential conflict of interest.

Copyright (c) 2021 Huang, Liao, Liu, Zhong, Cai and Long. This is an open-access article distributed under the terms of the Creative Commons Attribution License (CC BY). The use, distribution or reproduction in other forums is permitted, provided the original author(s) and the copyright owner(s) are credited and that the original publication in this journal is cited, in accordance with accepted academic practice. No use, distribution or reproduction is permitted which does not comply with these terms. 